\title{
A Hierarchical Self-Assembly Route to "Polyplex-in-Hydrophobic-Core" Micelles for Gene Delivery
}

Sundiata Kly, Lucas J. Andrew, Erin G. Moloney, Yuhang Huang, Jeremy E. Wulff, Matthew G. Moffitt*

Department of Chemistry, University of Victoria, PO Box 1700 Stn CSC, Victoria, BC V8W 2Y2, Canada

\section{Supporting Information}

Sample static light scattering data for cwc determination; Number intensity distributions for bulk and microfluidic PIHC micelles from CONTIN analysis of DLS data; 2-week PIHC micelle size and poldispersity stability data; LSCFM images of DAPI/DiI-labelled PIHC micelles deposited from an aqueous dispersion without cells; cell viability data from MDA-MB-231 cells dosed with PIHC micelles; and a table of actual flow rates.

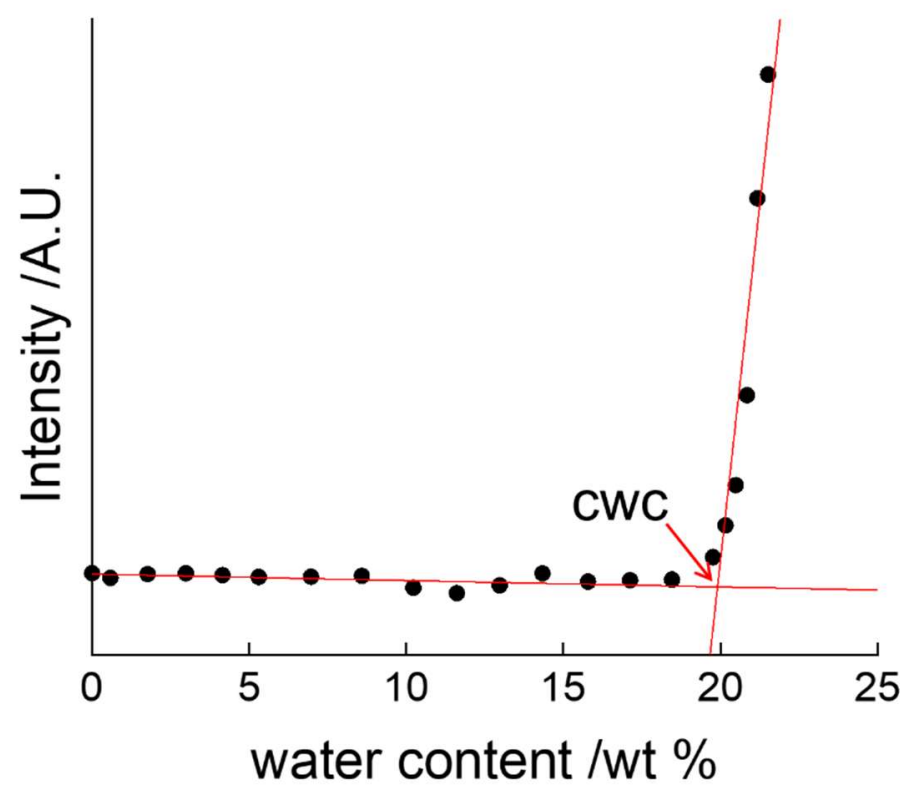

Figure S1. Critical water concentration (cwc) determination of PCL- $b$-PEG copolymer only (without PCL-pDNA) at the copolymer concentration and solvent composition of microfluidic PIHC micelle formation using static light scattering. Mean cwc and standard error $(19.2 \pm 0.3 \mathrm{wt}$ \%) were calculated from triplicate experiments; for each experiment, the cwc value was determined using linear regression of "baseline" and "rise" regions as shown. 


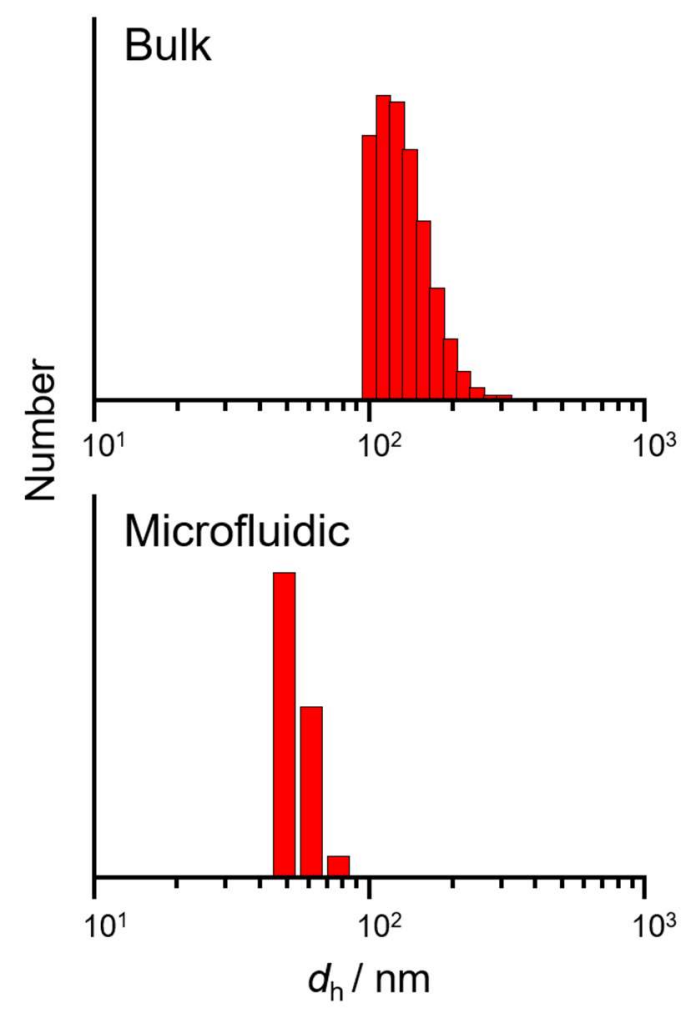

Figure S2. DLS number distributions (CONTIN analysis) of bulk (top) and microfluidic (bottom) PIHC micelle data shown in Figure 7B. In contrast to the intensity distribution of the microfluidic PIHC micelle sample (weighted by scattered light intensity) in which both smaller and larger particle populations are present (Figure 7B, bottom), only the smaller particle population is observed in the number distribution (bottom). 


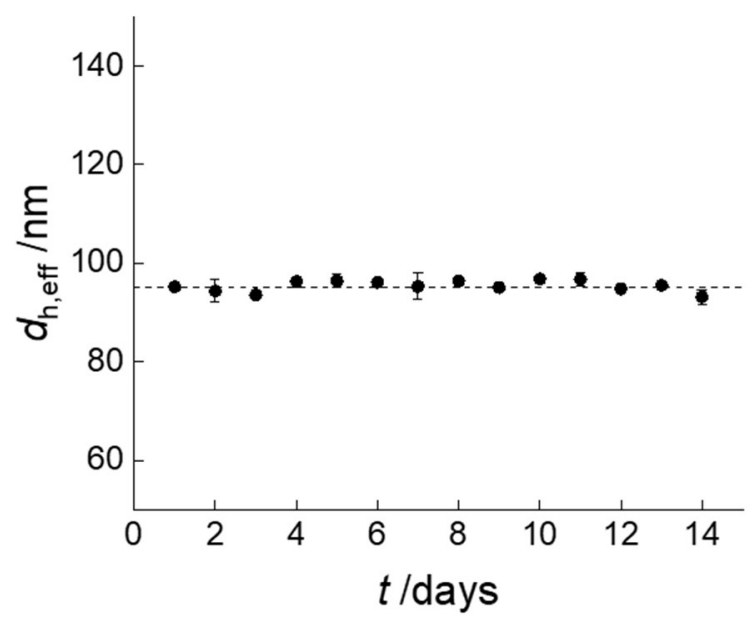

Figure S3. Size stability data for microfluidic PIHC micelle measured by DLS over two weeks for a single sample. The dashed horizontal line indicates the initial $t=1$ day measurement. Insignificant deviation from the initial size over 14 days indicates good stability over this time period.

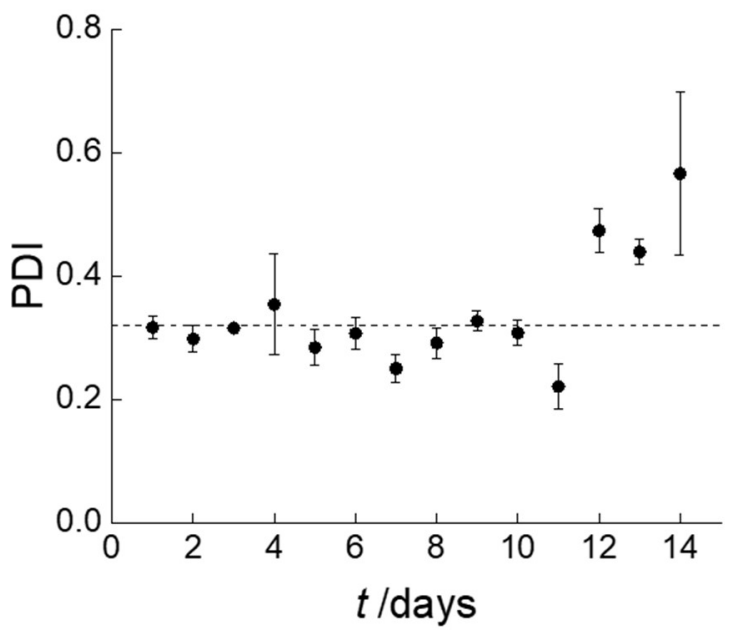

Figure S4. Poldispersity stability data for microfluidic PIHC micelle measured by DLS over two weeks for a single sample. The dashed horizontal line indicates the initial $t=1$ day measurement. Insignificant deviation from the initial polydispersity was observed over the first 12 days. 

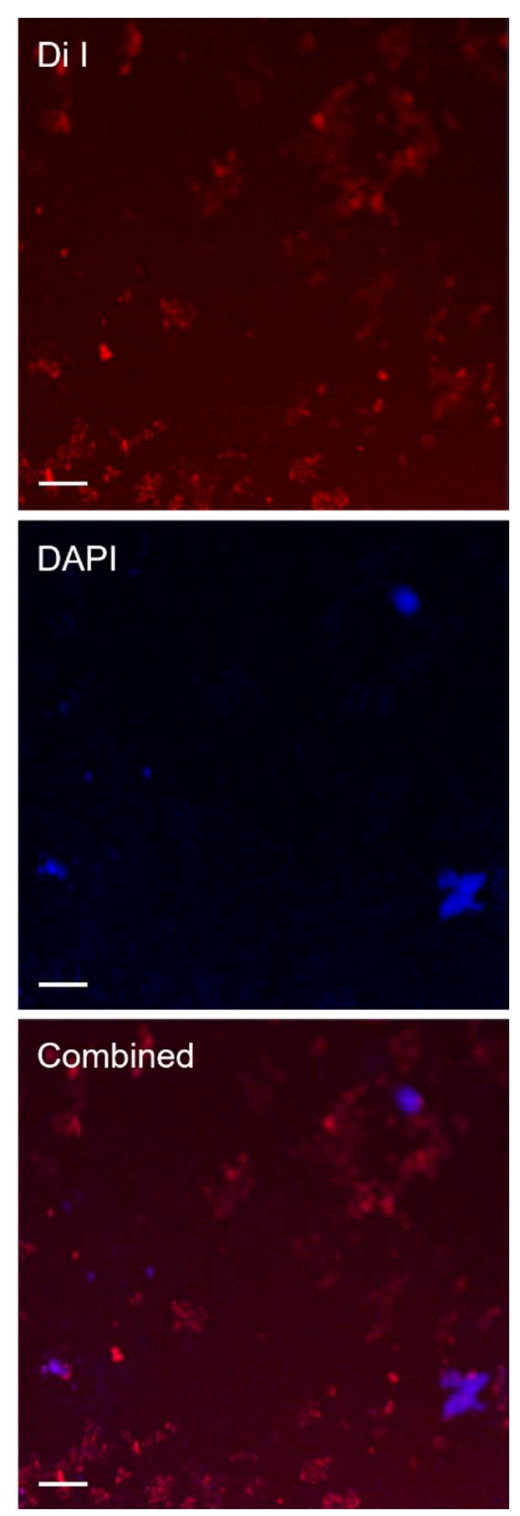

Figure S5. Fluorescence imaging data showing DAPI/DiI-labeled PIHC micelles deposited from an aqueous dispersion. Encapsulated pDNA was labelled with DAPI (blue) and PCL cores of the micelles were labelled with DiI (red). The red fluorescence channel (DiI) shows a greater number of fluorescent regions that the blue fluorescence channel (DAPI), indicating that some micelles do not contain pDNA. The overlay of red and blue channels (combined) shows co-localization between blue and red fluorescence, indicating that all pDNA is associated with micelles. Scale bars are $10 \mu \mathrm{m}$. 

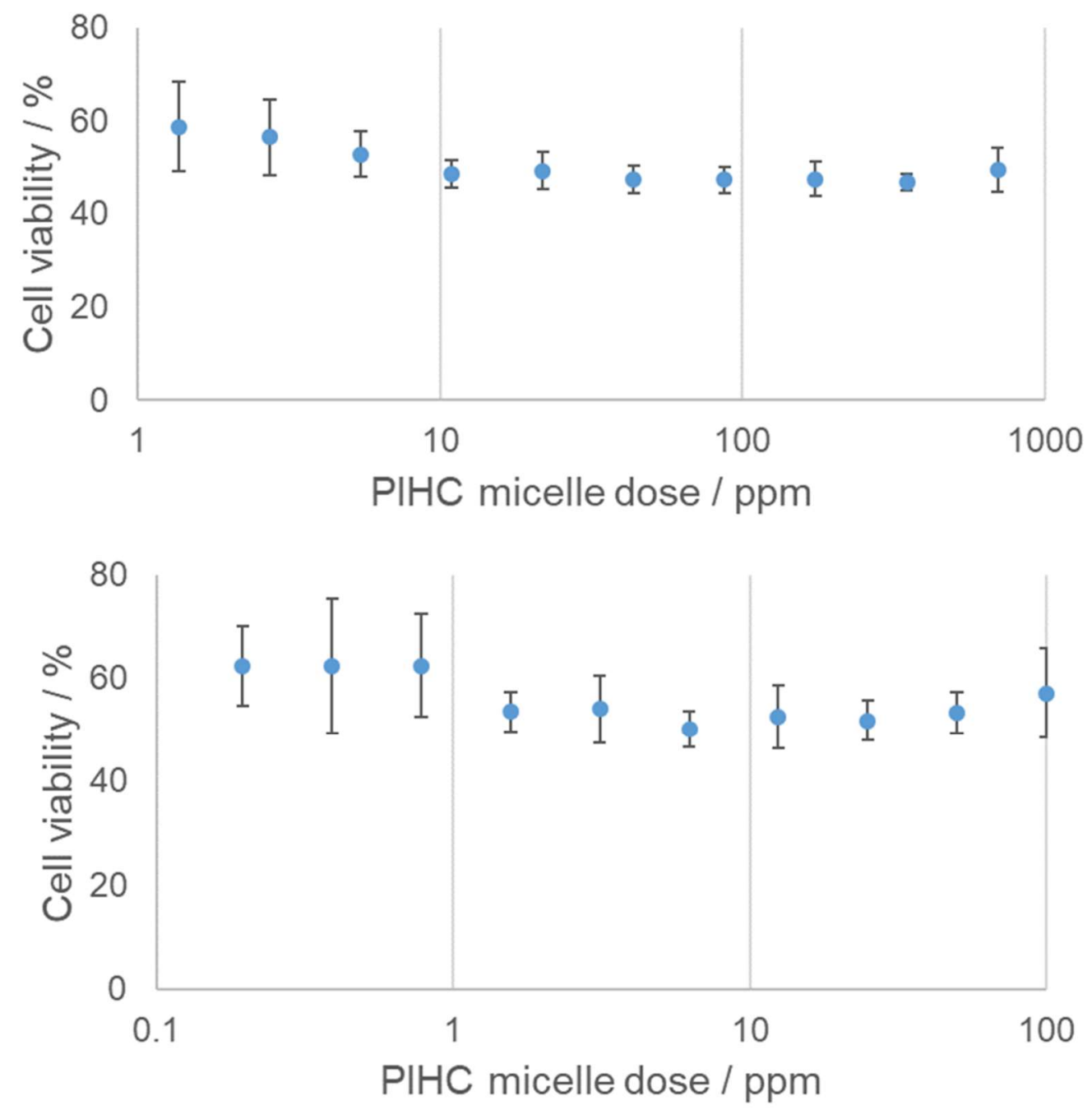

Figure S6. Cell viability of MDA-MB-231 cells with various dosing levels of PIHC micelles. Absence of dose dependence over 4 orders of magnitude of dosing levels indicates no cytotoxic effect of the PIHC micelles. 
Table S1. Actual Flow Rates of Various Preparations of PIHC Micelles within the Two-Phase Segmented Microfluidic Reactor Described in the Main Text.

\begin{tabular}{ccccc}
\hline PIHC micelles & Trial \# & $\begin{array}{c}\boldsymbol{Q}_{\text {gas }} \\
(\mu \mathrm{L} / \mathbf{m i n})\end{array}$ & $\boldsymbol{Q}_{\text {gas }} / \boldsymbol{Q}_{\text {liq }}$ & $\begin{array}{c}\boldsymbol{Q}_{\text {total }} \\
(\mu \mathrm{L} / \mathbf{m i n})\end{array}$ \\
\hline Figure 7A & 1 & 103 & 1.03 & 203 \\
& 2 & 100 & 1.00 & 200 \\
& 3 & 105 & 1.05 & 205 \\
\hline Figure 7B, 8, 9, 10 & 1 & 108 & 1.08 & 208 \\
& 2 & 106 & 1.06 & 206 \\
\hline Figure 11 & 3 & 102 & 1.02 & 202 \\
\hline
\end{tabular}

a) The nominal flow rate for all experiments was $200 \mu \mathrm{L} / \mathrm{min}$.

b)The liquid flow rate was assumed to be the sum of the three flow rate readings set on the syringe pump before running. 